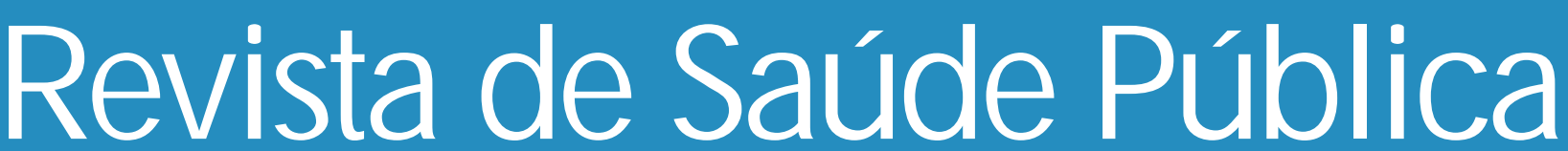

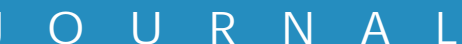

$0 \mathbf{F}$

$\mathbf{P} \mathbf{U}$

$B \quad L \quad$ I C

H E A L T H

\title{
U so da aspiração manual a vácuo na redução do custo e duração de intemamentos por aborto incompleto em Fortaleza, CE, Brasil
}

\section{Adoption of manual vacuum aspiration for treatment of incomplete abortion reduces costs and duration of patient's hospital stay in an urban area of N ortheastern Brazil}

Walter Fonseca, Chizuru Misago, Lucília Fernandes, Luciano Correia e Dirlene Silveira Departamento de Saúde Comunitária da U niversidade Federal do Ceará. Fortaleza, CE Brasil (W.F., L.C.); M aternal and Child Epidemiology U nit, London School of Hygiene and Tropical Medicine. London, U nited Kingdom (C.M.); Instituto sobre a Saúde da Mulher, Criança e Adolescente. Fortaleza, CE - Brasil (L.F.); Secretaria de Saúde do Estado do Ceará. Fortaleza, CE - Brasil (D.S.) 


\title{
U so da aspiração manual a vácuo na redução do custo e duração de intemamentos por aborto incompleto em Fortaleza, CE, Brasil*
}

\section{Adoption of manual vacuum aspiration for treatment of incomplete abortion reduces costs and duration of patient's hospital stay in an urban area of Northeastern Brazil}

\author{
Walter Fonseca, Chizuru Misago, Lucília Fernandes, Luciano Correia e D irlene Silveira \\ Departamento de Saúde Comunitária da U niversidade Federal do Ceará. Fortaleza, CE - Brasil (W.F., \\ L.C.); M aternal and Child Epidemiology U nit, London School of Hygiene and Tropical Medicine. \\ London, U nited Kingdom (C.M.); Instituto sobre a Saúde da Mulher, Criança e Adolescente. \\ Fortaleza, CE - Brasil (L.F.); Secretaria de Saúde do Estado do Ceará. Fortaleza, CE - Brasil (D.S.)
}

\begin{abstract}
Resumo
Introdução

O uso de aspiração a vácuo $(\mathrm{AV})$ no tratamento do aborto incompleto é prática bastante difundida em países desenvolvidos. Vários estudos nesses países indicam que o uso da técnica de aspiração manual a vácuo (AMV) pode conservar recursos do sistema de saúde e melhorar a qualidade do tratamento do aborto. No Brasil, o uso da AMV é procedimento de rotina nos hospitais e clínicas privados. Entretanto, na maioria dos hospitais da rede pública é utilizada somente a técnica de dilatação e curetagem (D\&C).
\end{abstract}

Metodologia

Foram utilizados métodos de avaliação rápida para estimar a variação do custo médio do tratamento e duração da estadia hospitalar, em um grupo de 30 pacientes admitidas com aborto incompleto em hospital público de Fortaleza, CE (Brasil). Participantes foram alocadas, randomicamente, em um dos dois grupos de tratamento investigados (AMV ou D\&C).

Resultados e Conclusões Os resultados sugerem que o uso da AMV, em substituição a D\&C, no trata-
mento do aborto incompleto, pode reduzir em até $41 \%$ o custo médio do trata-
mento e em $77 \%$ o tempo médio de hospitalização. Recomenda-se a realização
de estudos confirmatórios, como também que se aprofunde os conhecimentos
sobre a percepção do aborto e seu tratamento por parte do pessoal de saúde e da
população feminina.

Aborto, economia. Custos hospitalares.

* Financiado pela "Toyota Foundation", Tóquio, Japão.

Correspondência para/Correspondence to: Walter Fonseca - Rua Silva Jatay, 15 - sala 801 - 60165-070 Fortaleza, CE - Brasil. E-mail: pcreche@ufc.br

Recebido em 18.7.1996. Reapresentado em 17.2.1997. Aprovado em 21.5.1997. 


\section{Introduction}

Methodology

Results and Conclusion

\begin{abstract}
In most developed countries vacuum aspiration has been shown to be safer and less costly than sharp curettage (SC) for uterine evacuation. In many of the developing countries, including Brazil, sharp curettage (SC) is the most commonly used technique for treating cases of incomplete abortion admitted to hospital. The procedure often involves light to heavy sedation for pain control and an overnight hospital stay for patient recuperation and monitoring. Two hypotheses are examined: the first, that the use of manual vacuum aspiration (MVA) - a variation of the vacuum aspiration, would be less costly than SC for the treatment of cases of incomplete abortion admitted to hospital; and the second, that the treatment of incomplete abortion with MVA would substantially reduce the length of hospital stay.
\end{abstract}

Thirty women with diagnosis of first trimester incomplete abortion were randomly allocated to the SC or MVA group. Rapid-assessment data collection techniques were used to identify factors that contributed to cost reduction and hospital stay.

The results of the study show that, overall, patients treated for incomplete abortion with MVA spent $77 \%$ less time in the hospital and consumed $41 \%$ fewer resources than similarly diagnosed patients treated with SC. Recommendations are made as to the need of certain changes in patient management. Particularly necessary is information regarding cultural perception and concepts of abortion treatment.

Abortion. Hospital costs.

\section{INTRO DU ÇÃO}

No Brasil, assim como na maioria dos países em desenvolvimento, é cada vez mais acentuada a demanda por serviços da rede pública de saúde, contraposto pela contenção de custo daqueles que financiam o sistema. Torna-se, assim, imprescindível que os gestores dos serviços de saúde identifiquem estratégias para a utilização mais eficaz dos recursos existentes, sejam eles humanos, materiais ou financeiros $^{5}$. O presente artigo trata da redução dos custos do tratamento de abortos incompletos admitidos em hospitais da rede pública, tema de alta relevância em saúde pública.

A indução do aborto é legalmente permitida no Brasil somente quando necessária para salvar a vida da mulher ou quando a concepção ocorreu a partir de estupro (Código Penal, Artigos 126-129, Decreto-Lei n ${ }^{\circ} 2848$ de 7 de dezembro e emendas em 1941 e 1969). Entretanto, estimativas indiretas indicam que aproximadamente 1,4 milhões de abortos são provocados ilegalmente a cada ano no País ${ }^{2}$, revelando a necessidade urgente da reforma do Código Penal e revisão das estratégias de planejamento familiar atualmente em vigor no País ${ }^{10,13,19}$.

O tratamento do aborto incompleto, provocado ou espontâneo, em hospitais públicos, é responsável pela utilização de parte substancial de recursos do sistema de saúde, em países da América Latina ${ }^{3,4,9,}$ ${ }^{11}$. Dados de pesquisas e levantamentos feitos em hospitais identificam as complicações por aborto incompleto como uma das quatro principais causas de internação hospitalar no País, ${ }^{6,7,10}$.

Resultados de estudos em três Estados brasileiros revelam que é bastante comum entre as mulheres em idade fértil, das diferentes classes sociais, a prática do aborto provocado como último recurso para a interrupção de uma gravidez não planejada e não desejada ${ }^{6,7,10,20}$. Dados de serviço da maior maternidade pública de Fortaleza mostram que o número de admissões por complicações relacionadas ao aborto incompleto, provocado ou espontâneo, nos últimos cinco anos, mais do que triplicou. Esta tendência progressiva não foi acompanhada por uma elevação proporcional do número total de admissões aos serviços de gineco-obstetrícia ${ }^{6}$.

Nos hospitais públicos do Ceará, como na maioria dos hospitais públicos de outros Estados, o método usado para tratamento cirúrgico do aborto incompleto do primeiro trimestre é a dilatação e curetagem (D\&C). O uso da aspiração manual à vácuo (AMV) ou da aspiração elétrica intrauterina está, salvo raras exceções, restrito a clínicas e hospitais privados. Isto apesar das inúmeras evidências de pesquisas realizadas em países desenvolvidos que reportam ser a aspiração intrauterina à vácuo mais eficaz, mais se- 
gura e de menor custo se comparada à $\mathrm{D} \& \mathrm{C}$ no tratamento do aborto incompleto ${ }^{15,16,1824}$. Estudos recentes, em hospitais de dois países em desenvolvimento, documentaram redução de até $50 \%$ no custo médio do tratamento e na duração da estadia hospitalar, em pacientes com aborto incompleto, a partir da introdução do $\mathrm{AMV}^{15}$.

O presente estudo buscou investigar a redução do custo médio do tratamento e duração da estadia hospitalar, em pacientes admitidas com aborto incompleto em hospital público em Fortaleza, a partir da introdução da AMV. Com isto, pretende-se trazer novos subsídios que possam contribuir para a melhoria do tratamento do aborto incompleto em hospitais públicos no País.

\section{METO DO LOG IA}

Fortaleza, local do estudo, com cerca de 2 milhões de habitantes, Capital do Estado do Ceará, Nordeste do Brasil, tem na pesca, comércio e turismo a base de sua economia. Aproximadamente um terço da população vive em favelas, $65 \%$ dos domicílios dispõe de água tratada e $25 \%$ de rede de esgotos. A prestação de serviços em saúde, na quase sua totalidade, é feita de forma gratuita através de cerca de 70 centros de saúde, 7 postos de saúde e 36 hospitais. A cobertura vacinal no primeiro ano de vida é bastante elevada ( $85 \%$ ) e o coeficiente de mortalidade infantil é estimado em 50/1.000 nascidos vivos. Cerca de 35\% das mães não fazem pré-natal regularmente, e somente $45 \%$ das mulheres em idade reprodutiva fazem uso adequado de métodos de planejamento familiar. Mais de $95 \%$ dos nascimentos ocorrem em hospital ${ }^{21,22}$.

O presente estudo foi conduzido entre 11 de dezembro de 1995 e 4 de janeiro de 1996, em uma maternidade pública de referência de Fortaleza. Nessa maternidade, são admitidas, mensalmente, uma média de 200 mulheres para tratamento de aborto incompleto. É procedimento de rotina do serviço de emergência, o tratamento do aborto incompleto com a D\&C, realizada por médicos e residentes. Somente a partir de julho de 1994, através de convênio estabelecido entre a Secretaria de Saúde do Estado do Ceará e o "International Projects Assistance Services" [IPAS], a técnica da AMV vem sendo introduzida nos serviços de gineco-obstetrícia da maternidade estudada*. A técnica da AMV tem sido utilizada no tratamento de casos de aborto incompleto, sem complicações graves e com menos de 13 semanas de gestação. No período de três meses anteriores ao início do estudo, uma média mensal de $20(10 \%)$ casos de aborto incompletos foi tratada com AMV. A grande maioria das mulheres admitidas por aborto incompleto (90\%), foi tratada com a D\&C.

Métodos de avaliação rápida foram utilizados para a identificação de fatores que contribuem na qualidade, eficiência e custo do tratamento do aborto incompleto admi- tido na maternidade. Apesar da natureza qualitativa dos métodos de avaliação rápida, eles também agregam informações que podem ser quantificadas, como estimativas de custo e tempo de duração do tratamento do aborto. Os métodos de avaliação rápida mostram-se especialmente eficientes quando usados para examinar tema ou prática que é específico, bem definido, e sujeito a pouca variação, como a prática de tratar aborto incompleto de primeiro trimestre em ambientes hospitalares ${ }^{15,23}$. Os procedimentos médicos utilizados no tratamento do aborto, exceto em casos de complicações graves, são padronizados, e os mesmos recursos básicos são utilizados no tratamento de todas as pacientes ${ }^{15}$. Estudos realizados em países desenvolvidos revelam diferenças importantes na comparação do custo e tempo de hospitalização de pacientes tratadas com a D\&C ou AMV. Isto justificaria o uso de técnicas de avaliação rápida ao invés do recrutamento de um número elevado de participantes a serem acompanhadas prospectivamente. Assim, dada a especificidade do tema investigado e por ser a rotina de tratamento do aborto uma área de domínio do pessoal do hospital, a observação por um curto espaço de tempo de uma amostra de quinze mulheres em cada grupo foi considerada adequada para os objetivos do presente trabalho. Estudos recentes sobre o tratamento hospitalar do aborto incompleto, realizados no Kênia, México e Bangladesh, utilizando a mesma metodologia, indicam também que amostragens contendo entre dez e quinze pacientes, em cada grupo estudado, podem ser consideradas suficientes para o tipo de avaliação proposta $^{15,16}$.

Restringiu-se a participação no estudo às mulheres com até 12 semanas de gestação e que não apresentavam complicações graves relacionadas ao aborto incompleto, tais como, hemorragia grave, septicemia ou evidências de procedimento invasivo, com perfuração ou corpos estranhos na vagina e/ ou útero. As pacientes selecionadas segundo os critérios de inclusão referidos, foram alocadas, randomicamente, em um dos dois grupos de tratamento, D\&C e AMV.

Duas enfermeiras, com experiência em pesquisa em saúde reprodutiva e familiarizadas com a rotina de serviço da maternidade, foram recrutadas como entrevistadoras. Antes do início do procedimento cirúrgico, as pacientes eram informadas por uma das entrevistadoras sobre o objetivo da pesquisa e o seu consentimento para participação no estudo obtido. Foi assegurado às pacientes a confidencialidade das informações coletadas. Não houve recusa de participação em qualquer dos grupos.

Foram utilizados métodos qualitativos e quantitativos de pesquisa, incluindo a observação de pacientes, desde a admissão ao serviço de emergência até o término do procedimento cirúrgico com a D\&C ou AMV. Informações de alta hospitalar, foram obtidas a partir dos prontuários médicos. Foi utilizado formulário estruturado, baseado em modelo sugerido pelo IPAS ${ }^{1}$, na coleta de dados relativos ao tempo dispendido com cada procedimento médico, custo de drogas empregadas no tratamento, incluindo analgesia e sedação, assim como na estimativa parcial do 
custo com cada paciente, durante a hospitalização. Informações adicionais sobre o tratamento dos casos de aborto incompleto, recrutados para o estudo, foram obtidas através de entrevistas com pessoal médico e técnico-administrativo do hospital. Foram incluídos, para o cálculo de custos da AMV, os preços de cânulas e seringas. Os custos de material reutilizável, tais como tesouras, velas de Hegar, curetas, foco cirúrgico e outros, gastos com desinfecção e esterilização do material utilizado, bem como outros custos indiretos relacionados com a hospitalização, não puderam ser estimados. No tratamento do aborto com D\&C participaram um médico e/ou residente, um anestesista e uma auxiliar de enfermagem, e no tratamento com AMV um médico e/ou residente, uma enfermeira e uma auxiliar de enfermagem.

O software Epi-Info versão $6.0^{8}$ foi utilizado na preparação e análise dos dados coletados. Foram estimados o tempo e custo médio de hospitalização para cada um dos grupos, com a utilização da tabela de análise de variância na comparação das médias dos dois grupos. Este teste cujos resultados têm distribuição $\mathrm{F}$ é equivalente ao teste $\mathrm{t}\left[\mathrm{F}=\mathrm{t}^{2}\right]^{8,17}$.

\section{RESULTAD O $S$}

A Figura 1 apresenta o tempo médio (em horas) de hospitalização das pacientes, para cada uma das três fases do tratamento, de acordo com o tipo de procedimento cirúrgico utilizado. O tempo médio de permanência no hospital para as mulheres submeti-

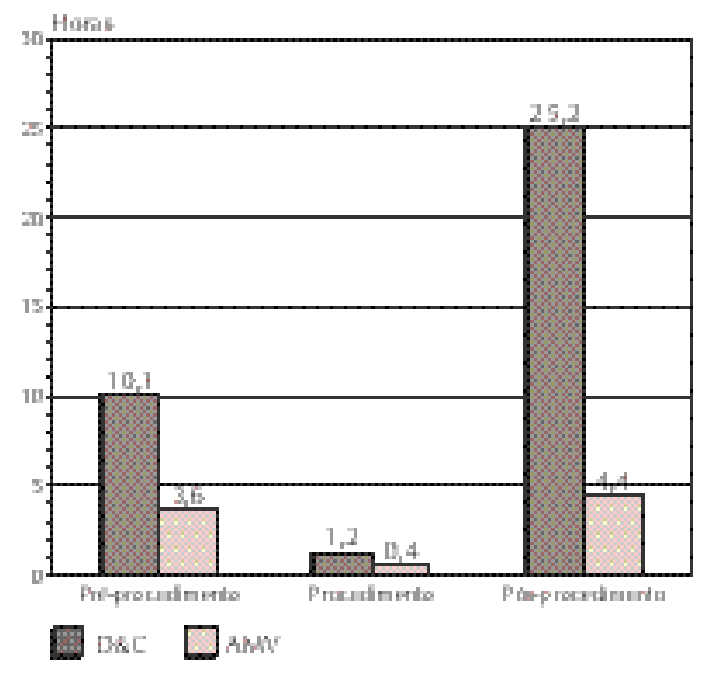

Figura 1 - Tempo médio (em horas) de hospitalização do aborto, para cada fase do tratamento, de acordo com o tipo de procedimento realizado. Fortaleza, 1996.

Figure 1 - Average duration (in hours) of hospitalization due to abortion/miscarriage, for each phase of treatment, according to the kind of procedure undertaken. Fortaleza, 1996. das à D\&C ou AMV foi de 36,5 e 8,4 horas, respectivamente. Pode-se observar uma redução de $77 \%$ no tempo médio de hospitalização para as pacientes tratadas com a AMV, em comparação com o grupo de pacientes submetidas à $\mathrm{D} \& \mathrm{C}(\mathrm{p}<0,001)$. Em todas as fases do tratamento, a AMV apresentou nítida redução do tempo médio dispensado por paciente, durante a hospitalização. A média de espera da paciente para a realização da $\mathrm{D} \& \mathrm{C}$ foi de 10,1 horas, enquanto para pacientes tratadas com a AMV foi de apenas 3,6 horas, indicando uma redução de $65 \%$ no tempo médio de espera $(\mathrm{p}<0,001)$. Em relação ao tempo médio necessário para a realização do procedimento cirúrgico, observou-se, mais uma vez, que a $D \& C$ requereu três vezes mais tempo que a AMV ( 1,2 versus 0,4 horas, $\mathrm{p}<0,001)$. Diferenças ainda mais acentuadas foram verificadas em relação ao tempo de permanência no hospital após o procedimento cirúrgico. Entre as mulheres tratadas com a AMV o tempo médio de permanência no hospital, durante a fase "pós-procedimento" (4,4 horas), foi cerca de seis vezes menor do que no grupo de mulheres submetidas à $\mathrm{D} \& \mathrm{C}$ ( 25,2 horas), o que representa uma redução de $83 \%(\mathrm{p}<0,001)$.

A Tabela mostra a estimativa de custos relativos aos salários da equipe médica encarregada do tratamento da paciente, por fase do procedimento cirúrgico, segundo o método utilizado. O custo médio com salários para a realização da D\&C e da AMV foi de $\mathrm{R} \$ * 3,55$ e $\mathrm{R} \$ * 4,13$, respectivamente. Assim, observa-se um aumento no custo da AMV em relação a D\&C de 14\% ( $<<0,001)$. Entretanto, em relação aos gastos com drogas, intrumentos e materiais utilizados no tratamento do aborto, a AMV apresentou uma redução de $66 \%$ ( $\mathrm{p}<0,001)$. O custo relacionado a este item foi estimado em $\mathrm{R} \$ * 13,15$ para a $\mathrm{D} \& \mathrm{C}$ e $\mathrm{R} \$ *$ 5,81 para as pacientes tratadas com a AMV.

Tabela - Custo médio (em reais)* com salários, drogas, instrumentos e material utilizados para cada procedimento. Fortaleza, 1996.

Table - Average cost (in reais) including salario, drugs, instruments and materials used for each procedure. Fortaleza, 1996.

\begin{tabular}{lrl}
\hline & D\&C & AMV \\
\hline Salários & 3,55 & 4,13 \\
$\begin{array}{l}\text { Drogas, instrumentos e } \\
\text { outros materiais }\end{array}$ & 13,15 & 5,81 \\
\hline Total & 16,70 & 9,94 \\
\hline
\end{tabular}

* O valor da unidade do Real foi estimado com base no Dólar vigente na época (US\$ $1.00 \approx \mathrm{R} \$ 1,00$ ). 


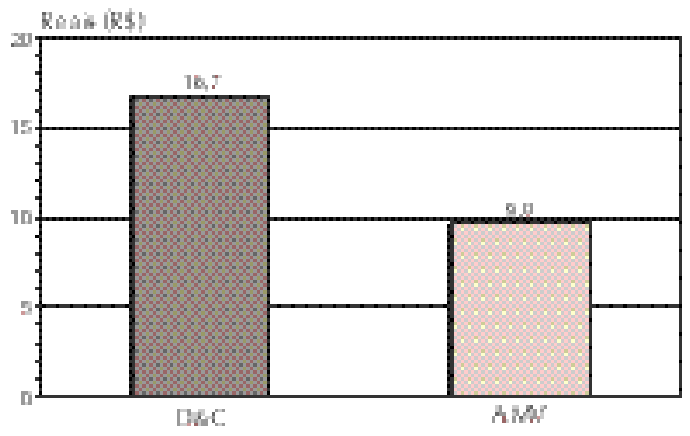

Figura 2 - Custo médio do procedimento por complicação de aborto, por paciente, de acordo com método utilizado. Fortaleza, 1996.

Figure 2 - Average cost of proceeding for complications arising from abortion/miscarriage, per patient, according to the method used. Fortaleza, 1996.

O custo médio por paciente, durante a internação hospitalar, de acordo com o método utilizado, é apresentado na Figura 2. Foram incluídos somente os custos com salários, drogas, materiais e instrumentos. Pacientes submetidas ao tratamento com a D\&C acusaram um custo total, em média, de $\mathrm{R} \$ * 16,70$, enquanto a média do custo do tratamento do aborto com a AMV foi de $\mathrm{R} \$ *$ 9,90. Observa-se, portanto, uma redução de $41 \%$ no custo médio do tratamento das pacientes que utilizaram a AMV, em comparação com pacientes tratadas com a $\mathrm{D} \& \mathrm{C}(\mathrm{p}<0,001)$.

\section{DISCU SSÃO}

Os resultados do estudo mostram que a substituição da D\&C pela AMV, no tratamento do aborto incompleto do primeiro trimestre, reduz substancialmente, tanto o custo total do tratamento, como o tempo de permanência das mulheres no hospital. No grupo de mulheres tratadas com a AMV, houve uma redução de $41 \%$ no custo médio do internamento, em relação às mulheres que foram submetidas à D\&C. Este achado confirma resultados de pesquisas recentes, realizadas no México e Kênia ${ }^{15}$.

No presente estudo, as estimativas de custo da D\&C e AMV não refletem o real custo destes serviços, que devem ser muito superiores aos aqui apresentados, uma vez que, por razões operacionais, não foi possível incluir vários itens relacionados ao tratamento e estadia hospitalar. Optou-se, então, por utilizar alguns itens diretamente relacionados ao custo do tratamento que permitissem comparar os dois métodos investigados. Assim, o estudo não incluiu os custos indiretos associados com uma estadia hospitalar, tais como lavanderia, alimentação, energia e outros. A inclusão desses itens aumentaria, sobretudo, a estimativa de custo da D\&C, que apresentaram tempo de estadia hospitalar muito superior ao das mulheres tratadas com AMV. Deve-se ainda considerar que a estimativa de custos da D\&C não incluiu gastos com equipamentos reutilizáveis, como velas de Hegar, curetas e outros. Entretanto, para o cálculo do custo médio de pacientes tratadas com a AMV foram computados os custos de cânulas e seringas. Assim, é esperado que, em média, a redução no custo do tratamento com a AMV, em comparação com a $\mathrm{D} \& \mathrm{C}$, seja de magnitude ainda superior à apresentada no presente estudo.

Em relação ao tempo médio de estadia no hospital, a redução observada foi também bastante acentuada. Mulheres tratadas com a AMV permaneceram, em média, pouco mais de oito horas no hospital, enquanto mulheres tratadas com a D\&C apresentaram um tempo médio de estadia hospitalar de aproximadamente 36 horas. Isto representou uma notável redução de $77 \%$ no tempo médio de hospitalização para as pacientes tratadas com AMV, em comparação com o grupo de pacientes submetidas a D\&C. Este resultado coincide com os achados de estudos recentes conduzidos em países em desenvolvimento ${ }^{15}$.

As características da AMV explicam essa notável redução de custos e estadia hospitalar, em relação à D\&C. As medidas para o controle da dor com o método AMV envolvem a sedação leve, analgesia e/ou anestesia local, realizadas pelo médico ginecologista encarregado do procedimento cirúrgico. A D\&C requer nível mais alto de medicamentos para o controle da dor, incluindo sedação intensa e analgesia, administrados sob supervisão de um médico anestesista. Isto resulta no aumento de custos relacionados à utilização de drogas, tempo de estadia hospitalar, notadamente no período de recuperação (pósprocedimento), e à necessidade da presença do anestesista. Ao mesmo tempo, o uso de níveis mais baixos, mas adequados, de controle da dor com a AMV, pode reduzir o risco de complicações relacionadas com a anestesia ${ }^{12}$.

O uso dos métodos de aspiração intrauterina são bastante conhecidos, e amplamente utilizados em serviços de saúde privados do País. Em países desenvolvidos, esses métodos já fazem parte da rotina de tratamento do aborto incompleto há várias décadas $^{12,15}$. Nesses países, as principais razões para a 
adoção desta prática médica foram a eficácia de praticamente $100 \%$ do método, associada a um índice de complicacões muito menor do que o existente com a D\&C. Também importante foi a observação de uma notável redução no custo do tratamento e no período de estadia no serviço de saúde, em comparação com a $\mathrm{D \& C} \mathrm{C}^{12,14}$. São desconhecidas as razões pelas quais, no Brasil, a D\&C é prática médica estabelecida e altamente institucionalizada no tratamento do aborto incompleto, em detrimento do uso da aspiração intrauterina.

Foi recentemente concluído, em Fortaleza, estudo utilizando metodologia qualitativa sobre a percepção do aborto e seu tratamento. Foram entrevistados técnicos de saúde da maternidade estudada e mulheres com aborto incompleto tratadas com AMV, e que reportaram aborto prévio tratado com a D\&C*. A análise preliminar dos dados revelam a boa aceitação do método AMV por parte dos técnicos de saúde do hospital e das mulheres participantes no estudo. Entre as principais vantagens do método AMV foram referidas a redução na duração da estadia hospitalar e a qualidade da assistência oferecida. Os resultados dessa, como também da presente investiga-

\section{REFERÊN CIAS BIBLIO G RÁFICAS}

1. ABERBATHY, M. et al. Guia para avaliar o uso de recursos para o tratamento do aborto incompleto, Carrboro, International Projects Assistance Services (IPAS), 1993.

2. ALAN GUTTMACHER INSTITUTE. Aborto clandestino: uma realidade latino-americana. Nova York, The Alan Guttmacher Institute, 1994.

3. BAYLEY, E.P. et al. A hospital study of illegal abortion in Bolívia. Bull. Pan. Am. Health Organ., 11:27-41, 1988.

4. BEMFAM. Pesquisa sobre a saúde familiar no Nordeste do Brasil - 1991. Rio de Janeiro, Sociedade Civil Bem-Estar Familiar no Brasil, 1992.

5. BITTAR, O.J.N. Produtividade em hospitais de acordo com alguns indicadores hospitalares. Rev. Saúde Pública, 30:5360, 1996.

6. COELHO, H.L.L. et al. Misoprostol and illegal abortion in Fortaleza, Brazil. Lancet, 341:1261-3, 1993.

7. COSTA, S. \& VESSEY, M.P. Misoprostol and illegal abortion in Rio de Janeiro, Brazil. Lancet, 341:1258-61, 1993. ção, servirão para apoiar o programa de atenção à saúde da mulher "Viva Mulher", da Secretaria de Saúde do Ceará, na elaboração de programas de treinamento para a introdução da técnica da AMV nos serviços de saúde de todo o Estado.

Em conclusão, os resultados do presente estudo sugerem que o uso da AMV em substituição a D\&C, no tratamento do aborto incompleto, pode reduzir significativamente o custo médio do tratamento e a duração da estadia hospitalar. Esses achados estão de acordo com a literatura existente, e têm relevância para o planejamento no setor de saúde. Estudos complementares são necessários para confirmar e ampliar estes resultados, como também para que se aprofunde os conhecimentos sobre a percepção do aborto por parte do pessoal de saúde e da população feminina.

\section{AGRADECIMENTOS}

À direção e aos funcionários do Hospital Geral César Cals por toda colaboração prestada no desenvolvimento do estudo. À Dra. Clarice Mota pelos comentários.
8. DEAN, A.G. et al. Epi Info, Version 6.0: a Word Processing Database and Statistics Program for Epidemiology on Microcomputers. Atlanta, Center for Disease Control and Prevention, 1994.

9. FIGA-TALAMANCA, I. Illegal abortion: an attempt to assess its costs to the health services and its incidence in the community. Int. J. Health. Serv., 16:375-89, 1986.

10. FONSECA, W. et al. Determinantes do aborto provocado entre mulheres em hospitais em localidade da região Nordeste do Brasil. Rev. Saúde Pública, 30:13-8, 1996.

11. FORTNEY, J.A. The use of hospital resources to treat incomplete abortions: examples from Latin America. Public. Health. Rep., 96:574-9, 1981.

12. GREENSLADE, F.C. et al. Manual vacuum aspiration: a summary of clinical and programmatic experience worldwide. Carrboro, International Projects Assistance Services, 1993.

13. HARDY, E. \& REBELLO, I. La discusión sobre el aborto provocado en el Congresso Nacional Brasileño: el papel del movimento de mujeres. Cad. Saúde Pública, 12:259-66, 1996.

* Comunicação pessoal de C. Misago, Maternal and Child Epidemiology Unit, London School of Hygiene and Tropical Medicine, London, United Kingdom. 
14. HENSAHAW, S.K. Indiced abortion: a world review. Fam. Plan. Perspect., 22:76-89, 1990.

15. JOHNSON, B.R. et al. Cost and resource utilization for the treatment of incomplete abortion in Kenya and Mexico. Soc. Sci. Med., 36:1443-53, 1993.

16. KAY, B. \& KABIR, S.M. A study of costs and behavioral outcomes of menstrual regulation services in Bangladesh. Soc. Sci. Med., 26:597-604, 1988.

17. KIRKWOOD, B. The essential of medical statistics. London, Blackwell Scientific Publications, 1988.

18. KIZZA, A. P. M. \& ROGO, K. Assessment of the Manual Vacuum Aspiration (MVA) equipment in the management of incomplete abortion. East Afr. Med.J., 67:812-21, 1990.

19. MISAGO, C. et al. Características médicas do aborto provocado entre mulheres admitidas em hospitais de Fortaleza. Rev.Matern.Assis Chateaubriand, 2:9-12, 1995.
20. SCHOR, N. Investigação sobre ocorrência de aborto em pacientes de hospital de um centro urbano do Estado de São Paulo, Brasil. Rev. Saúde Pública, 24:144-51, 1990.

21. SECRETARIA DE SAÚDE DO ESTADO. Aspectos socioeconômicos dos municípios do Ceará, 1994/95. Fortaleza, Instituto de Planejamento do Ceará, 1996.

22. SECRETARIA DE SAÚDE DO ESTADO. Indicadores de saúde materno-infantil no Ceará, 1994. Fortaleza, 1994.

23. SCRIMSHAW, S.C.M. \& HURTADO, E. Rapid assessment procedures for nutrition and primary health care: anthropological approaches to improving programme effectiveness. Tokyo, UCLA/The United Nations University, 1987. ( Latin American Center Reference Series, 11).

24. TIETZE, C. \& LEWIT, S. Joint program for the study of abortion (JPSA): early medical complications of legal abortion. Stud. Fam. Plan., 3:97-119, 1972. 